\title{
Magnetic Alignment of Electrospun Fiber Segments Within a Hydrogel Composite Guides Cell Spreading and Migration Phenotype Switching
}

\author{
Harrison L. Hiraki ${ }^{1}$, Daniel L. Matera ${ }^{2}$, Michael J. Rose ${ }^{3}$, Robert N. Kent', \\ Connor W. Todd ${ }^{3}$, Mark E. Stout ${ }^{3}$, Anya E. Wank ${ }^{3}$, Maria C. Schiavone ${ }^{3}$, \\ Samuel J. DePalma', Alexander A. Zarouk ${ }^{1}$ and Brendon M. Baker ${ }^{1,2 *}$

\begin{abstract}
' Department of Biomedical Engineering, University of Michigan, Ann Arbor, MI, United States, ${ }^{2}$ Department of Chemical Engineering, University of Michigan, Ann Arbor, MI, United States, ${ }^{3}$ Department of Mechanical Engineering, University of Michigan, Ann Arbor, MI, United States
\end{abstract}

OPEN ACCESS

Edited by:

Luca Cristofolini,

University of Bologna, Italy

Reviewed by:

Prakriti Tayalia,

Indian Institute of Technology

Bombay, India

Sônia Maria Malmonge,

Federal University of ABC, Brazil

${ }^{*}$ Correspondence:

Brendon M. Baker

bambren@umich.edu

Specialty section:

This article was submitted to Biomaterials,

a section of the journal

Frontiers in Bioengineering and

Biotechnology

Received: 11 March 2021 Accepted: 10 May 2021

Published: 16 June 2021

Citation:

Hiraki HL, Matera DL, Rose MJ, Kent RN, Todd CW, Stout ME, Wank AE, Schiavone MC,

DePalma SJ, Zarouk AA and Baker BM (2021) Magnetic Alignment of Electrospun Fiber Segments Within

a Hydrogel Composite Guides Cell

Spreading and Migration Phenotype

Switching.

Front. Bioeng. Biotechnol. 9:679165.

doi: 10.3389/fbioe.2021.679165
Fibrous extracellular matrix (ECM) proteins provide mechanical structure and adhesive scaffolding to resident cells within stromal tissues. Aligned ECM fibers play an important role in directing morphogenetic processes, supporting mechanical loads, and facilitating cell migration. Various methods have been developed to align matrix fibers in purified biopolymer hydrogels, such as type I collagen, including flow-induced alignment, uniaxial tensile deformation, and magnetic particles. However, purified biopolymers have limited orthogonal tunability of biophysical cues including stiffness, fiber density, and fiber alignment. Here, we generate synthetic, cell-adhesive fiber segments of the same length-scale as stromal fibrous proteins through electrospinning. Superparamagnetic iron oxide nanoparticles (SPIONs) embedded in synthetic fiber segments enable magnetic field induced alignment of fibers within an amorphous bulk hydrogel. We find that SPION density and magnetic field strength jointly influence fiber alignment and identify conditions to control the degree of alignment. Tuning fiber length allowed the alignment of dense fibrous hydrogel composites without fiber entanglement or regional variation in the degree of alignment. Functionalization of fiber segments with cell adhesive peptides induced tendon fibroblasts to adopt a uniaxial morphology akin to within native tendon. Furthermore, we demonstrate the utility of this hydrogel composite to direct multicellular migration from MCF10A spheroids and find that fiber alignment prompts invading multicellular strands to separate into disconnected single cells and multicellular clusters. These magnetic fiber segments can be readily incorporated into other natural and synthetic hydrogels and aligned with inexpensive and easily accessible rare earth magnets, without the need for specialized equipment. 3D hydrogel composites where stiffness/crosslinking, fiber density, and fiber alignment can be orthogonally tuned may provide insights into morphogenetic and pathogenic processes that involve matrix fiber alignment and can enable systematic investigation of the individual contribution of each biophysical cue to cell behavior. 


\section{INTRODUCTION}

Stromal extracellular matrix (ECM) provides manifold biophysical cues that direct both physiologic and pathologic cell behavior. A major component of stromal ECM are fibrous proteins (e.g., collagens, fibronectin, and elastin) that serve as cell-adhesive scaffolding and provide structural and mechanical support to a variety of tissues (Poltavets et al., 2018). Cells dynamically deposit, reorganize, and respond to the fibrous architecture of the ECM (Dallas et al., 2006). Through contact guidance cues, fibrous protein structures direct a variety of morphogenetic processes including tenogenesis, branching morphogenesis, and angiogenesis (Kirkpatrick et al., 2007; Brownfield et al., 2013; Iannone et al., 2015). Fibrous proteins are also heavily implicated in initiating and directing invasion from primary tumors during breast cancer progression (Provenzano et al., 2006). Second harmonic generation (SHG) imaging has provided valuable insights into collagen architecture during morphogenesis and disease progression (Ingman et al., 2006). With this insight, biomaterials recapitulating aligned fibrous architectures have been developed to model and direct such processes in vitro.

Purified biopolymers such as type I collagen and fibrin have been used to model stromal ECM as both possess fibrous topography. However, polymerization of these materials under typical conditions produces hydrogels with isotropic fibrous architecture due to the stochastic nature of fibrillogenesis (Wakuda et al., 2018). To better model highly anisotropic fibrous architecture, such as that found in tendons and around primary breast tumors, several approaches to align collagen fibers have been developed. Early methods to align collagen gels took advantage of the slight negative charge of collagen to align fibers with powerful magnetic fields (Dickinson et al., 1994). More recently, a diversity of methods to align collagen gels have emerged including flow-induced alignment (Han et al., 2016), embedding of magnetic particles which are dragged through the gel with an external magnetic field (Guo and Kaufman, 2007; Provenzano et al., 2008; Carey et al., 2016; Taufalele et al., 2019), application of tensile forces via stretching (Riching et al., 2015), and fibroblastmediated reorganization of fibers (Dumont et al., 2013; Ray et al., 2017). These methods create highly anisotropic collagen gels and have been instrumental in investigating how aligned fibrous architecture influences cell behavior. However, purified biopolymers typically have limited orthogonal control of relevant biophysical cues (Wolf et al., 2009; Mason et al., 2013). For example, increasing type I collagen gel concentration leads to commensurate increases in fiber density, stiffness, and ligand density. In contrast, synthetic hydrogels (e.g., polyethylene glycol, methacrylated gelatin, and functionalized polysaccharides) offer enhanced orthogonal tunability of these physical properties. However, these amorphous hydrogels typically lack fibrous architecture (Baker and Chen, 2012; Li et al., 2017).

Electrospinning offers a means to generate fibrous topography that closely recapitulates the geometry and length-scale of fibrous proteins found in stromal ECM. The electrospinning process uses a voltage gradient to draw solid fibers from a charged polymer solution. Previous work with PVA, PLGA, and dextran methacrylate has shown that cell migration on electrospun, synthetic fiber matrices captures key aspects of cell migration in fibrous natural ECM proteins like type I collagen (Kim et al., 2016; Wang et al., 2018; Hong et al., 2019). Recent work from our group and others have established means to generate embedded fiber segments within amorphous synthetic hydrogels (Rose et al., 2017, 2018; Matera et al., 2019). These hydrogel composites enable cell studies within topographically complex fibrous environments in which fiber density and stiffness can be orthogonally controlled. However, such composites rely on encapsulating fiber segments within a hydrogel, resulting in a random distribution of embedded fibers.

In this work, we embedded superparamagnetic iron oxide nanoparticles (SPIONs) within our synthetic fiber segments during the electrospinning process to enable fiber alignment under an externally applied magnet field. Degree of fiber alignment within a 3D amorphous hydrogel proved sensitive to SPION density as well as the strength of the imposed magnetic field. Computational modeling of the magnetic field revealed dependence upon magnet placement, where hydrogels appropriately positioned during crosslinking can achieve homogeneous alignment of constituent fibers. We find that fiber length influences the frequency of entanglement as a function of fiber density during magnetic alignment. Finally, we demonstrate the use of magnetic fiber alignment within our hydrogel composite system to control encapsulated tendon fibroblast (tenocyte) alignment and elicit directional migration of a breast epithelial cell line. Interestingly, we find that fiber alignment not only biases epithelial cell migration direction, but also promotes cell-cell breakage events leading to a switch in 3D migration phenotype.

\section{MATERIALS AND METHODS}

\section{Reagents}

All reagents were purchased from Sigma Aldrich and used as received, unless otherwise stated.

\section{Synthesis of Modified Dextran Vinyl Sulfone \\ Dextran Vinyl Sulfone (DVS)}

Dextran was functionalized with vinyl sulfone pendant groups using a previously described protocol (Matera et al., 2019). Briefly, linear high molecular weight dextran (MW 86,000 Da; MP Biomedicals) was reacted with pure divinyl sulfone (Fisher) under basic conditions ( $\mathrm{pH}$ 13.0). Functionalization was terminated through $\mathrm{pH}$ adjustment to 5.0 with hydrochloric acid. Reaction products were dialyzed against milli- $\mathrm{Q}$ water for 3 days, with water changed twice daily. Purified products were then lyophilized for 3 days and reconstituted at $100 \mathrm{mg} \mathrm{mL}^{-1}$ in a Michael-type addition buffer (MTAB; $1 \mathrm{~N} \mathrm{NaOH}, 1$ M HEPES, $1 \mathrm{mg} \mathrm{mL}^{-1}$ phenol red in milli-Q water). 


\section{DVS Fiber Segment Fabrication}

DVS was dissolved at $0.6 \mathrm{~g} \mathrm{~mL}^{-1}$ in a $1: 1$ mixture of milli-Q water and dimethylformamide. SPIONs with or without polyvinylpyrrolidone (PVP) coating (US Research Nanomaterials) were added at 2.5, 5, or $10 \mathrm{w} / \mathrm{v} \%$. Lithium phenyl-2,4,6-trimethylbenzoylphosphinate (LAP) photoinitiator $(10 \mathrm{v} / \mathrm{v} \%)$ and methacrylated rhodamine $(2.5 \mathrm{v} / \mathrm{v} \%)$ (Polysciences, Inc.) were added to the solution to facilitate photoinitiated crosslinking and fluorescent visualization, respectively. Polymer solutions were electrospun in a humiditycontrolled glove box held at $21^{\circ} \mathrm{C}$ and $30-35 \%$ relative humidity. Electrospinning was performed at $0.25 \mathrm{~mL} \mathrm{hr}^{-1}$ flow rate, $7 \mathrm{~cm}$ gap distance, and $-9.0 \mathrm{kV}$ voltage onto a grounded copper collective surface. Fibers were collected on glass cover slides and crosslinked under ultraviolet light $\left(100 \mathrm{~mW} \mathrm{~cm}^{-2}\right)$ for $20 \mathrm{sec}$. Custom fabricated chrome photomasks were placed over fiber mats during UV photocrosslinking to control fiber length. Fiber mats were detached from cover slides into milliQ water and broken into individual fiber segments. Fiber segments were purified through a series of centrifugation steps to remove uncrosslinked polymer and entangled fibers before resuspension in $\mathrm{MTAB}$ at $10 \mathrm{v} / \mathrm{v} \%$. Prior to encapsulation within bulk hydrogels, fibers were coupled with $2.0 \mathrm{mM}$ RGD (CGRGDS; CPC Scientific) via Michael-type addition to enable eventual cell adhesion.

\section{Magnetic Gelation Chamber Fabrication}

The magnet housing apparatus was created with solid $1 / 2^{\prime \prime}$ aluminum and $1.00^{\prime \prime} \times 2.00^{\prime \prime}$ T-slotted aluminum. Two N52 neodymium magnets (K\&J Magnetics) were housed in carriages made from solid aluminum, securing the magnets on either side with a hole removed at the face of the magnet. The carriages were attached to the T-slotted aluminum rails on the base of the setup, which aligned the carriages and allowed them to controllably slide along the rails with a crankshaft. A 3D printed clamp was designed to hold a petri dish containing fiberreinforced hydrogel composites within the center of the magnet axis. To prevent encapsulated fibers and cells from settling during hydrogel crosslinking, an Arduino-controlled stepper motor with a $3 \mathrm{D}$-printed grip was programmed to flip the petri dish $180^{\circ}$ every $20 \mathrm{~s}$.

\section{Computation Visualization of Magnet Field Lines}

COMSOL Multiphysics software was used to quantify magnetic flux densities and visualize field lines between magnets. A 3D stationary study modeling magnetic fields generated without current (permanent magnet) was performed. Two cylinders $(1.905 \times 3.81 \mathrm{~cm})$ modeling the two magnets were placed within a $15-25 \mathrm{~cm}$ radius sphere to model air impedance. Surface flux density of the two cylinders was set to $661.9 \mathrm{mT}$, the innate surface field of N52 neodymium magnets. A single slice in the $\mathrm{X}-\mathrm{Z}$ plane was generated to visualize magnetic flux density around and between the magnets. White arrows were overlaid to visualize magnetic field lines with the arrow size logarithmically proportional to strength of magnet flux along the field line. To generate $1 \mathrm{D}$ plots of magnetic flux density in the Z-direction across various magnet spacings, flux density values were extracted from a path along the magnet axis.

\section{Hydrogel Formation and Fiber Alignment}

DVS gels were formed via an analogous click reaction at 3.1 w/v\% with $9.64 \mathrm{mM}$ VPMS cross-linker and heparin binding peptide $(2 \mathrm{mM})$. All hydrogel precursor solutions were made in PBS. To create fibrous hydrogels, a defined stock solution $(10 \mathrm{v} / \mathrm{v} \%)$ of suspended fiber segments in MTAB was mixed into hydrogel precursor solutions prior to gelation. Via controlling the dilution of the fiber suspension, fiber density was tuned at a constant hydrogel weight percentage and bulk stiffness. Hydrogel precursor solutions were injected into $5 \mathrm{~mm}$ in diameter polydimethylsiloxane gaskets and crosslinked at $37^{\circ} \mathrm{C}$ for $1 \mathrm{~h}$. To align encapsulated fibers, hydrogels were crosslinked between the two magnets of magnetic gelation chamber.

\section{Cell Lines and Culture}

\section{Primary Tenocyte Harvesting}

Mouse resident tenocytes were harvested with a previously established protocol (Shimada et al., 2014). Briefly, mouse tail tendons were encapsulated in $2 \mathrm{mg} \mathrm{mL}^{-1}$ collagen I (Advanced Biomatrix), allowing cells to proliferate into the gel for 11 days. Gels were then digested with $0.25 \mathrm{mg} \mathrm{mL}^{-1}$ collagenase from C. histolyticum and cells were centrifuged out. Tenocytes were cultured in DMEM supplemented with $10 \mathrm{v} / \mathrm{v} \%$ fetal bovine serum (Fisher) and $1 \mathrm{v} / \mathrm{v} \%$ penicillin/streptomycin/amphotericin B. Tenocytes were passaged near confluency at a 1:2 ratio and used for studies until passage 3. For 3D hydrogel encapsulation studies, media was additionally supplemented with $50 \mathrm{ng} \mathrm{mL}^{-1} \mathrm{~L}$-ascorbic acid-2-phosphate and transforming growth factor- $\beta 3$ (Peprotech). Human mammary epithelial cells MCF10A (ATCC) were cultured in DMEM/F12 (1:1) supplemented with $5 \mathrm{v} / \mathrm{v} \%$ horse serum (Fisher), $20 \mathrm{ng} \mathrm{mL}-1$ rhEGF (Peprotech), $0.5 \mathrm{mg} \mathrm{mL}^{-1}$ hydrocortisone, $100 \mathrm{ng} \mathrm{mL}^{-1}$ cholera toxin, and $10 \mu \mathrm{g} \mathrm{mL}^{-1}$ insulin (Fisher). MCF10As were passaged at confluency at a 1:4 ratio and used for studies until passage 8. Spheroid formation: MCF10As were detached with $0.25 \%$ trypsin-EDTA (Life Technologies), counted, and formed into 200 cell-sized spheroids overnight in inverse pyramidal PDMS microwells (AggreWell ${ }^{\mathrm{TM}}$, Stem Cell Technologies) treated with $0.5 \%$ Pluronic F-127 to prevent cell adhesion. All cells were cultured at $37^{\circ} \mathrm{C}$ and $5 \% \mathrm{CO}_{2}$.

\section{Cytotoxicity Screens}

SPIONs with or without PVP coating were suspended in complete MCF10A media over a range of densities. To create SPION conditioned media, SPIONs were incubated in complete MCF10A media for $48 \mathrm{~h}$ and then centrifuged out at 20,000 rcf for $30 \mathrm{~min}$. 2D monolayer assay: MCF10A cells plated on glass coverslips were exposed to SPION containing media or SPION conditioned media for $12 \mathrm{~h}$ then incubated in serum free MCF10A media with Hoechst stain $(1 \mu \mathrm{g} / \mathrm{mL})$ and propidium iodide $(1 \mu \mathrm{g} / \mathrm{mL})$ for 20 min prior to fixing. 3D hydrogel assay: DVS fiber segments containing SPIONs with or without PVP coating were coencapsulated with single MCF10A cells $(1,000,000$ 
cells $\mathrm{mL}^{-1}$ ) in DVS hydrogels. After $12 \mathrm{~h}$ in culture, hydrogels were incubated in serum free MCF10A media with Hoechst stain $(2 \mu \mathrm{g} / \mathrm{mL})$ and propidium iodide $(2 \mu \mathrm{g} / \mathrm{mL})$ and incubated on a rocker plate at $0.33 \mathrm{~Hz}$ for $1 \mathrm{~h}$ to enhance diffusive transport prior to fixing.

\section{Single Cell Spreading Studies}

Primary-derived tenocytes $\left(5,000,000\right.$ cells $\left.\mathrm{mL}^{-1}\right)$ and fiber segments (3 v/v\%) were co-encapsulated in DVS hydrogels. Studies were maintained in complete tenocyte media for 7 days, with media replenished every other day.

\section{Spheroid Migration Studies}

MCF10A spheroids were harvested and centrifuged to remove residual single cells. Spheroids $\left(6000 \mathrm{~mL}^{-1}\right)$ and fiber segments ( $3 \mathrm{v} / \mathrm{v} \%)$ were co-encapsulated in DVS hydrogels. Studies were cultured in complete MCF10A media for 6 days, with media replenished every other day.

\section{Fluorescence, Staining, and Microscopy}

Samples were fixed with $4 \%$ paraformaldehyde for $1 \mathrm{~h}$ at room temperature. To visualize the actin cytoskeleton and nuclei, samples were stained with phalloidin and DAPI for $1 \mathrm{~h}$ at room temperature. For immunostaining, gels were additionally permeabilized in PBS containing Triton X-100 (5 v/v\%), sucrose $(10 \mathrm{w} / \mathrm{v} \%)$, and magnesium chloride $(0.6 \mathrm{w} / \mathrm{v} \%)$ and blocked in $4 \%$ BSA. Fluorescent imaging was performed with a Zeiss LSM 800 laser scanning confocal microscope. For migration analysis, Z-stacks were acquired with a 10x objective. All images are presented as maximum intensity projections.

\section{Cell Migration Analysis}

A previously established custom MATLAB image analysis code was used to extract morphometric data from spheroid migration studies (Hiraki et al., 2021). Briefly, max intensity projections of spheroid nuclei and F-actin channels were separately thresholded and object size filtered to remove background. A user-drawn ellipsoidal ROI covering the spheroid body was used to separate the spheroid body from migratory cells within outgrowths. The code segmented F-actin structures into individual outgrowths which were defined as either contiguous or non-contiguous based on contiguity with the spheroid body. A separate function segmented overlapping nuclei to identify all nuclei within outgrowths. Individual outgrowth F-actin masks were used to determine migration distance into the surrounding hydrogel utilizing a separate custom function. Corresponding individual nuclei masks were used to determine nuclei locations, nuclear counts, and mark non-contiguous outgrowths as either multicellular clusters or single cells. All individual outgrowth nuclei and F-actin masks were then summed to produce final images of nuclei and F-actin channels. Individual outgrowth nuclei and F-actin masks were saved with counted nuclei or plotted lengths, respectively, and assigned an index to address discrepancies or outliers within final quantified data. Resulting data were stratified by contiguity with the spheroid body and exported to a spreadsheet containing individual outgrowth indices, number of migratory cells, outgrowth areas, and migration distances. Finally, spheroid body and outgrowth masks were summed across all analyzed spheroids to produce heatmap overlays.

\section{Statistics}

Statistical significance was determined by one-way analysis of variance (ANOVA) with post hoc analysis (Tukey test), with significance indicated by $p<0.05$. All data are presented as mean \pm standard deviation.

\section{RESULTS}

\section{Fabrication of a Magnetically Responsive Electrospun Fiber Segments}

To create fiber segments on the same length-scale as fibrous proteins found in stromal ECM, DVS polymer solution was electrospun to produce fibers $\sim 2 \mu \mathrm{m}$ in diameter (Matera et al., 2019). Electrospun fiber mats were processed into suspensions of fiber segments which could then be encapsulated in $3 \mathrm{D}$ hydrogels and aligned by an externally applied magnetic field (Figure 1A). SPIONs added to the electrospinning solution were stably encapsulated within fibers upon photocrosslinking (Figure 1B, red arrowheads). To define the strength of an imposed magnetic field during hydrogel gelation, a magnetic gelation chamber was designed with adjustable spacing of two N52 neodymium permanent magnets (Figure 1C). The setup consisted of an aluminum base and rails upon which two magnet carriages housing the neodymium magnets could be controllably spaced with a crankshaft over a range of 6-20 cm. A hydrogel precursor solution containing DVS fiber segments was crosslinked within a petri dish positioned between the two magnets. To prevent fibers or cells from settling during hydrogel crosslinking, a clamp attached to an Arduino-controlled stepper motor flipped the petri dish $180^{\circ}$ within the magnetic field every 20 s during gelation (Figure 1D). This setup enabled facile control over fiber alignment via magnet spacing and resulting magnetic field strengths (Figure 1E).

\section{Computational Visualization of Magnetic Field Lines and Field Strength}

Magnetic field lines produced by a single permanent magnet resemble concentric ellipses radiating from the magnet's north to south pole. When opposite poles of two juxtaposed permanent magnets are aligned, field lines combine and densify as a function of spacing between the magnets. To determine the strength of the magnetic field produced within the magnetic gelation chamber, we modeled magnetic flux density using COMSOL. A 3D model of two permanent magnets was created by placing two cylinders of equivalent geometry within a sphere to model air impedance (Figure 2A). Surface fields of $669.1 \mathrm{mT}$ were set at the cylinder surfaces to model N52 neodymium boundary conditions. Magnet flux density along the major magnet axis (Z-axis) was determined across a range of magnet spacings to quantitate the magnetic field strength applied to centrally 


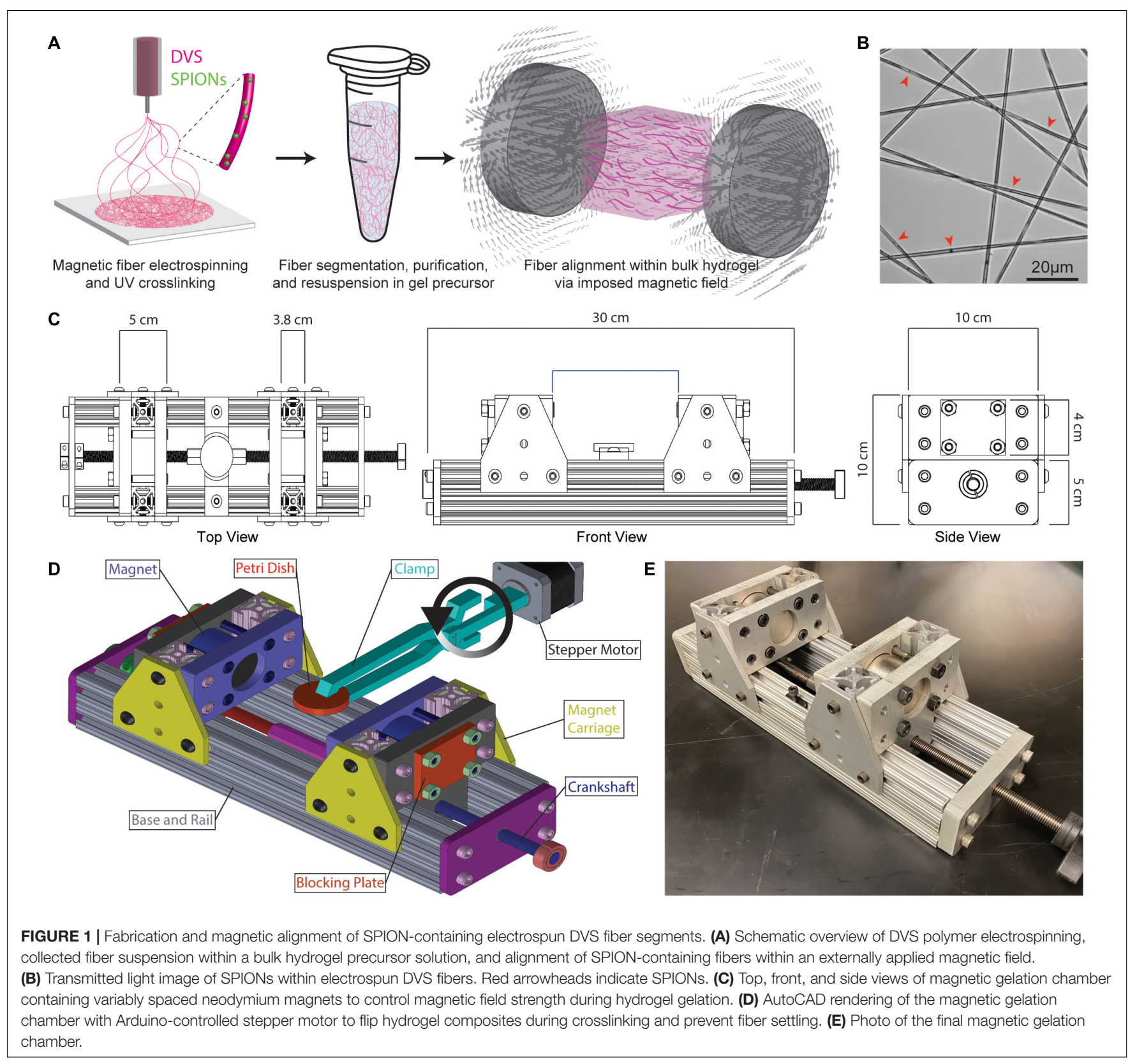

positioned hydrogels (Figure 2B). Flux density along the Z-axis was parabolic in strength, highest at the magnet surfaces and decaying exponentially to the center position between magnets $(Z=0 \mathrm{~cm})$. The smallest magnet spacing achievable $(6 \mathrm{~cm})$ produced a flux density of $126.9 \mathrm{mT}$ at the center. Increasing magnet spacing to 12 and $18 \mathrm{~cm}$ significantly decreased flux density to 24.8 and $7.5 \mathrm{mT}$, respectively. To better visualize field lines between magnets, flux density heat maps were generated and overlaid with white arrows logarithmically proportional to regional flux densities (Figure 2C). Field lines were parallel to magnet axis orientations and decayed exponentially once outside of the radius the magnets $(X<-1.905 \mathrm{~cm}$ or $\mathrm{X}>1.905 \mathrm{~cm}$ ). Thus, hydrogel composites positioned within the central region of the magnets are exposed to a nearly homogeneous magnetic field with field lines running parallel to the magnets' axes.

\section{Degree of Fiber Alignment Is Jointly Regulated by SPION Density and Magnet Spacing}

To optimize DVS fiber alignment, we first modulated the density at which SPIONs were incorporated into the DVS electrospinning solution. We noted a slight decrease in fiber segment yield with increasing SPION density (data not shown), likely due to the SPIONs interfering with the electrospinning process. Fiber segments were encapsulated in 3D DVS gels at $1 \mathrm{v} / \mathrm{v} \%$ and aligned at a magnet spacing of $6 \mathrm{~cm}$ (Figure 3A). 


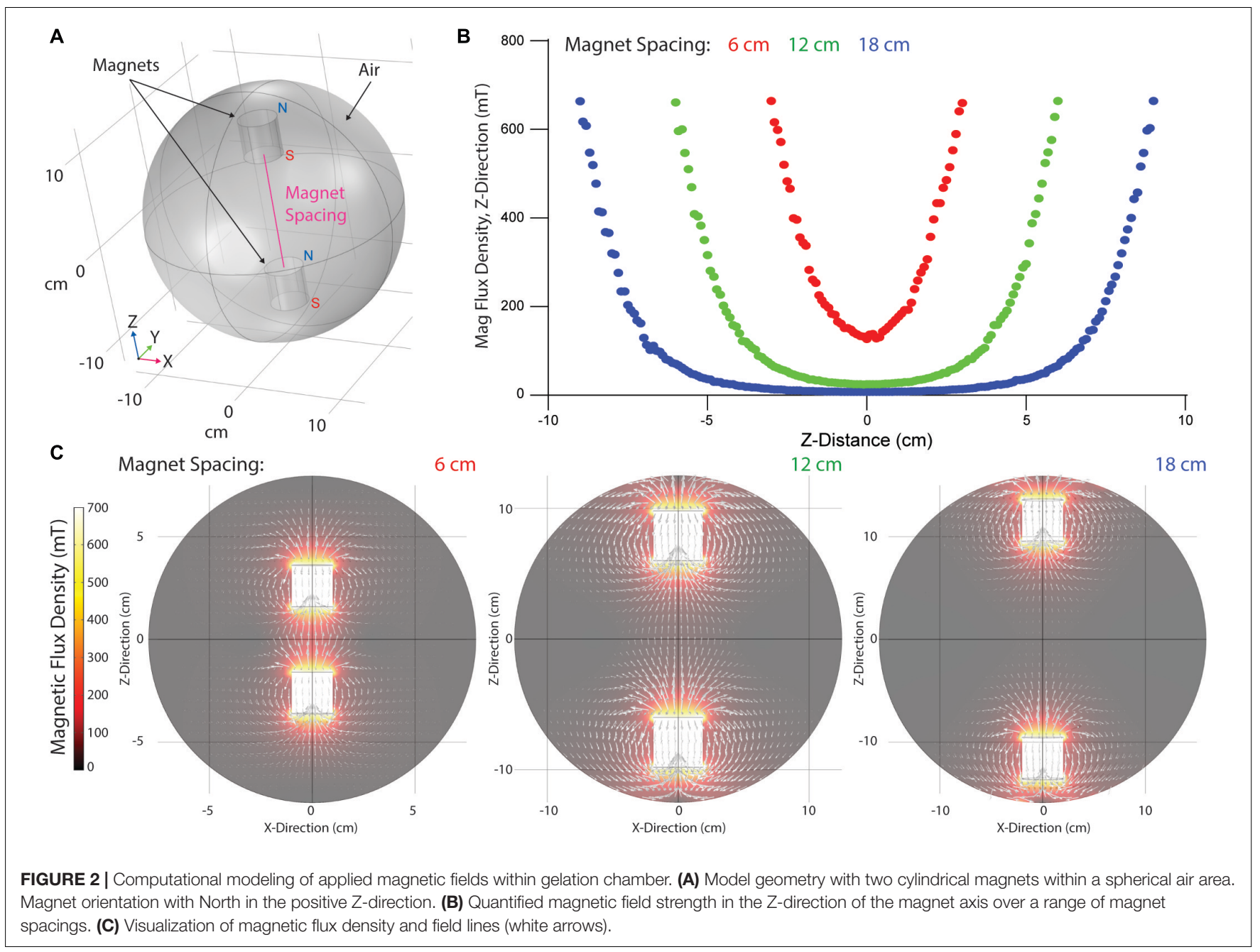

Degree of fiber alignment was quantified via anisotropy score generated with the ImageJ plugin FibrilTool (Boudaoud et al., 2014). Hydrogels containing fibers without SPIONs crosslinked at $6 \mathrm{~cm}$ magnet spacing resulted in randomly oriented fibers (Figure 3B; -SPION), indicating DVS fiber segments are not innately responsive to a magnetic field. Hydrogels containing fibers with the highest SPION density $\left(10 \mathrm{mg} \mathrm{mL}^{-1}\right)$ crosslinked outside of the magnetic gelation chamber also resulted in randomly oriented fibers (Figure 3B; -Mag), indicating SPIONcontaining fibers do not align in the absence of an external magnetic field. In contrast, hydrogels containing SPION fibers crosslinked within the magnetic field contained aligned fibers oriented in the direction of the magnetic field. The highest degree of fiber alignment resulted from a SPION density of $5 \mathrm{mg} \mathrm{mL}^{-1}$, suggesting a density of $2.5 \mathrm{mg} \mathrm{mL}^{-1}$ was below an optimal density required for magnetic forces to align fibers. Conversely, at $10 \mathrm{mg} \mathrm{mL}^{-1}$, SPIONs began to form large aggregates within the electrospinning solution, potentially decreasing the total amount retained in fiber segments and therefore limiting alignment (Gutiérrez et al., 2019). We next assessed alignment of $5 \mathrm{mg} \mathrm{mL} \mathrm{m}^{-1}$ SPION-containing fibers across a range of magnetic field strengths by varying the spacing between the two magnets (Figure 3C). A step-wise decrease in fiber alignment was observed with increasing magnet spacing (Figure 3D), indicating fiber segments can be aligned with field strengths between 5 and $125 \mathrm{mT}$ (Figure 2) and that the degree of alignment is a function of both SPION density and field strength. To further visualize degree of fiber alignment, we utilized OrientationJ to produce color map images based on fiber orientation for fibers aligned across SPION encapsulation densities and magnet distances (Supplementary Figure 1; Püspöki et al., 2016).

\section{Decreasing Fiber Length Prevents Entanglement at High Fiber Density}

Previous reports on approaches to align type I collagen gels have noted collagen fiber entanglement ( $\mathrm{Ng}$ and Swartz, 2003, 2006; Guo and Kaufman, 2007; Omidinia-Anarkoli et al., 2017). As a high fiber density is key to modeling fibrous tissues such as tendons and the stroma of breast tissue during cancer progression (Provenzano et al., 2006; Docheva et al., 2015), we next determined if increases in fiber density led to entanglement. Fiber density was modulated through the input fiber volume fraction of the hydrogel precursor solution over a range of 

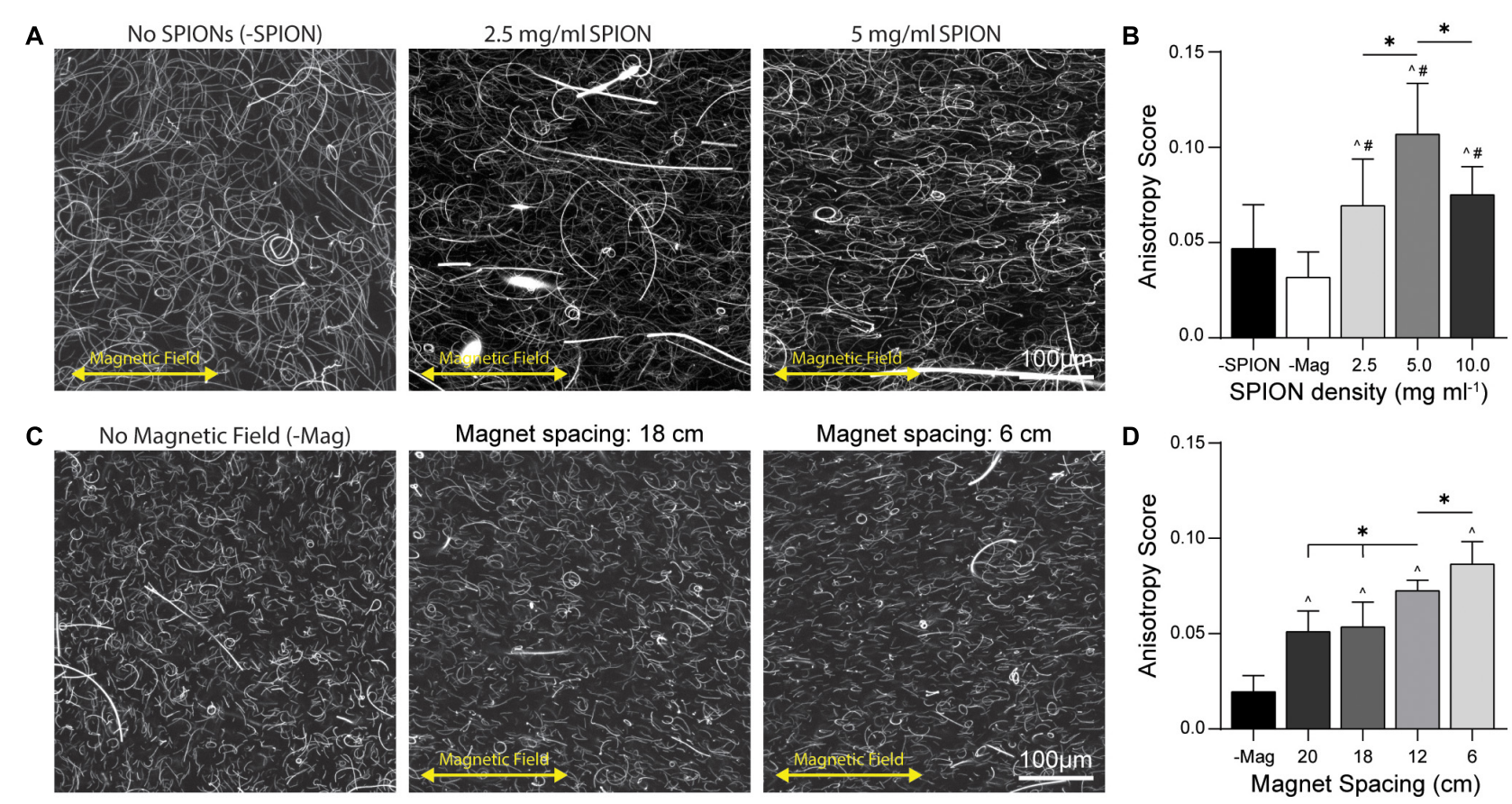

FIGURE 3 | Fiber alignment as a function of SPION density and magnet spacing. (A) Fiber alignment at $1 \mathrm{v} / \mathrm{V} \%$ fiber density in $3 \mathrm{D}$ DVS hydrogels across a range of encapsulated SPION densities at $6 \mathrm{~cm}$ magnet spacing. (B) FibrilTool quantification of anisotropic fiber alignment. (C) Fiber alignment of $5 \mathrm{mg}$ mL ${ }^{-1}$ SPION fibers at $1 \mathrm{v} / \mathrm{v} \%$ over a range of magnet spacings and (D) quantification of fiber alignment. All data presented as mean \pm standard deviation (SD); *indicates a statistically significant comparison with $p<0.05$; $^{\wedge}$ indicates significance against -Mag; \# indicates significance against -SPION.

$1-5 \mathrm{v} / \mathrm{v} \%$ and gels were crosslinked at a magnet spacing of $6 \mathrm{~cm}$. At fiber densities at or below $3 \mathrm{v} / \mathrm{v} \%$, highly anisotropic fiber alignment was achieved with minimal evidence of entanglement (Figures 4A,B). However, at $4 \mathrm{v} / \mathrm{v} \%$ fiber density, entanglement was apparent which led to a significant decrease in fiber alignment (Figure 4B). Within these gels, heterogeneously distributed regions of localized fiber alignment vs. entanglement were observed (Figure $\mathbf{4 A}$, green and red inserts). At $5 \mathrm{v} / \mathrm{v} \%$ fiber density nearly all fibers were entangled in large clumps, leading to an anisotropy score similar to non-aligned fibers (Figure 3). As fiber entanglement occurred in regions where long fiber segments were co-encapsulated in high proximity, we sought to maintain alignment at higher fiber densities by decreasing fiber segment length. To do so chrome photomasks were placed over electrospun fiber mats during photocrosslinking (Figure 4C). Photomasks with arrays of square patterns $(100,150$, or $250 \mu \mathrm{m})$ yielded fiber segments spanning $60-120 \mu \mathrm{m}$ in average length; in contrast, fibers generated without photomasking were on average $225 \mu \mathrm{m}$ in length with considerably larger variance (Figure 4D). To test if shorter fibers diminished entanglement despite high encapsulation density, $5 \mathrm{v} / \mathrm{v} \%$ fibrous hydrogels were crosslinked at $6 \mathrm{~cm}$ magnet spacing. Fibers created with the 100 and $150 \mu \mathrm{m}$ photomasks were highly aligned and showed little evidence of entanglement, while gels containing $250 \mu \mathrm{m}$ photomasked fibers possessed regions of entanglement similar to fibers generated without photomasking (Figure 4E). Despite the lack of evident entanglement in either hydrogel, gels containing $150 \mu \mathrm{m}$ photomasked fibers had a significantly higher anisotropy score compared to gels containing $100 \mu \mathrm{m}$ photomasked fibers. This difference is likely due to the influence of object length in FibrilTool's calculation of anisotropy score (Figure 4F). To determine if rigid boundaries locally influenced fiber alignment, we imaged a cross-section of a $5 \mathrm{~mm}$ cylindrical hydrogel composite containing $150 \mu \mathrm{m}$ photomasked fibers. No regional differences in fiber alignment at gel boundaries perpendicular or parallel to fiber alignment were observed, indicating that magnetic alignment overcame any flow-induced alignment along boundaries (Figure 4G). In sum, magnetic alignment of $5 \mathrm{mg}$ $\mathrm{mL}^{-1}$ SPION-containing fibers optimally sized by photomasking resulted in homogeneous alignment.

\section{SPION Encapsulation Within Fiber Segments Prevents Cytotoxic Interaction With Cells}

The presence of charged SPIONs has previously been reported to be cytotoxic (Rose et al., 2017; Patil et al., 2018). To determine if the SPIONs used here are cytotoxic, and to test if cytotoxicity results from direct interaction of SPIONs with cells versus changes in media ion concentrations due to the addition of SPIONs, we added SPIONs or SPION-conditioned media to MCF10A mammary epithelial cell monolayers. Cell death, assessed via staining with membrane-impermeable propidium iodide (PI), was SPION dose-dependent with 1 and $0.5 \mathrm{mg}$ $\mathrm{mL}^{-1}$ SPION concentration in media resulting in significant increases in cell death relative to controls (Figures 5A,B). 


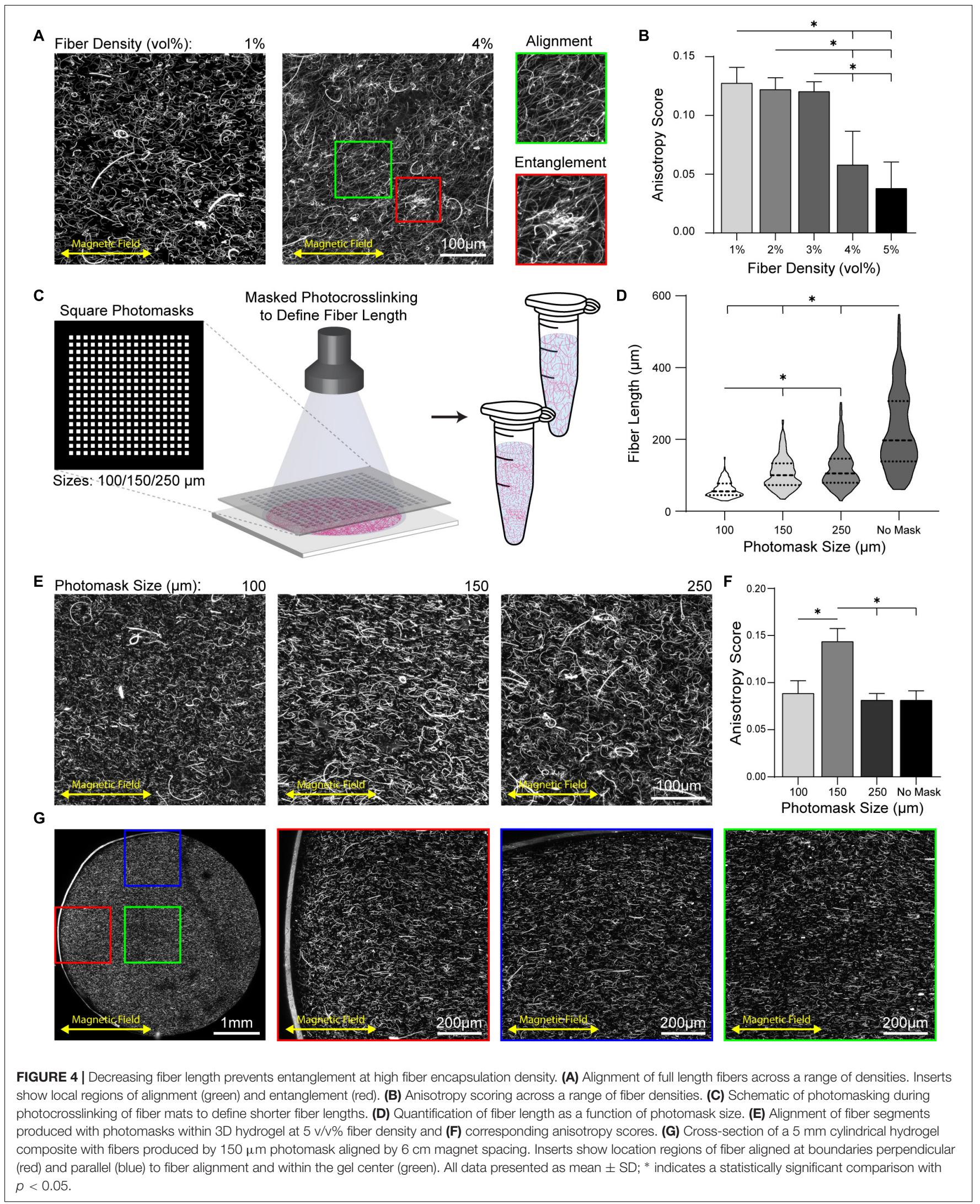


SPION-conditioned media at any concentration tested did not increase cell death above control levels, suggesting cytotoxicity resulted from direct cell interactions with SPIONs rather than changes in media ion concentrations. Polyvinylpyrrolidone (PVP) coating of biomaterials has previously been reported to reduce cytotoxicity (Zhi et al., 2013). As such, we repeated cytotoxicity experiments with PVP-coated SPIONs. A PVPcoated SPION dose-dependent increase in cell death was again observed, but cell death upon addition of $1 \mathrm{mg} \mathrm{mL}^{-1}$ SPIONs was lower with PVP coating. PVP-coated SPION-conditioned media did not induce cell death above control levels (Figure 5B). The significant decrease in cell death at the highest SPION concentration indicates PVP coating decreases cytotoxicity. However, it is worth noting the degree of cell death is minimal $(<3 \%)$ regardless of PVP coating. To assess cytotoxicity when SPIONs are embedded within fiber segments, we next coencapsulated single MCF10A cells and fibers containing $5 \mathrm{mg}$ $\mathrm{mL}^{-1}$ of SPIONs with or without PVP coating (Figure 5C). After $12 \mathrm{~h}$ in culture, no difference in cell death was observed, indicating insignificant SPION escape from fiber segments and limited cytotoxicity. Furthermore, no increase in cell death was observed in non-fibrous gels exposed to the strongest magnetic field (6 cm magnet spacing), indicating cells are not negatively affected by an externally applied magnetic field (Figure 5D). The ability to electrospin SPION-containing fibers or align fibers within 3D gels was not altered by PVP coating (Figure 5E), and therefore PVP-coated SPIONs were used in all subsequent studies.

\section{Fiber Alignment Directs Uniaxial Spreading in Primary Derived Mouse Tenocytes}

Alignment of fibrous ECM architecture is known to influence fibroblast spreading and polarization. For applications in tendon tissue engineering, alignment of tendon fibroblasts (tenocytes) within 3D hydrogels may be critical to mechanosensing and ECM deposition (Schoenenberger et al., 2018; Xu et al., 2021). To enable fibroblast adhesion to magnetic fibers, residual VS groups were functionalized with the cell-adhesive peptide, CGRGDS, via Michael-type addition. Primary tenocytes harvested from mouse tendons were co-encapsulated along with SPION-containing fibers in a bulk MMP-degradable DVS hydrogel to determine the influence of fiber alignment on tenocyte spreading and orientation. Magnet spacing was modulated to produce aligned $(6 \mathrm{~cm})$, partially aligned $(12 \mathrm{~cm})$, or non-aligned (no magnetic field) hydrogel composites. Tenocyte spreading in non-aligned gels included both stellate morphologies in which filopodia extended in all directions (Figure 6A, red arrowheads) and uniaxial spread morphologies with high aspect ratios (Figure 6A, yellow arrowheads). In contrast, tenocyte spreading in both partially aligned and aligned gels favored higher aspect ratios with the long axes of cells oriented in the direction of fiber alignment. Fiber alignment at the highest possible field strength $(6 \mathrm{~cm}$ magnet spacing) resulted in significantly more aligned cells than lower field strength (12 cm magnet spacing), as calculated

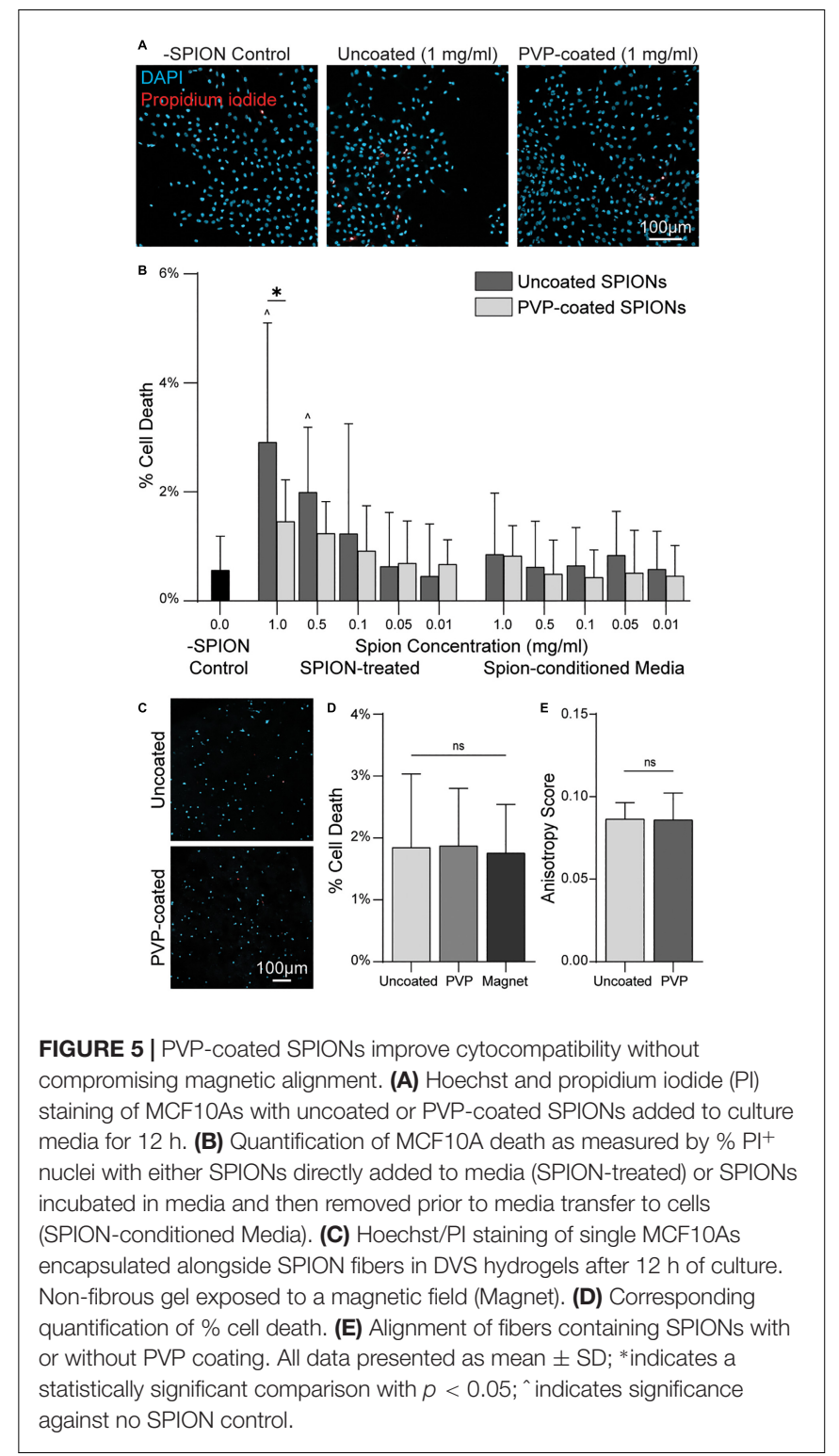

by the full width half max of cell orientation distributions (Figures 6B,C). Quantification of individual cell orientations revealed nearly random distributions in non-aligned gels. In partially aligned and aligned gels, the distribution of cell orientation increasingly narrowed with the majority of cells oriented within \pm 30 degrees of the fiber alignment axis (Figure 6D).

\section{Fiber Alignment Directs Multicellular Migration and Induces Migration Phenotype Switching}

ECM fiber alignment has also been heavily implicated in epithelial cell migration during transtromal escape from primary tumors. To examine the effect of fiber alignment on epithelial cell migration, MCF10A spheroids and SPION-containing fibers were co-encapsulated within MMP-degradable DVS gels. Degree 
A

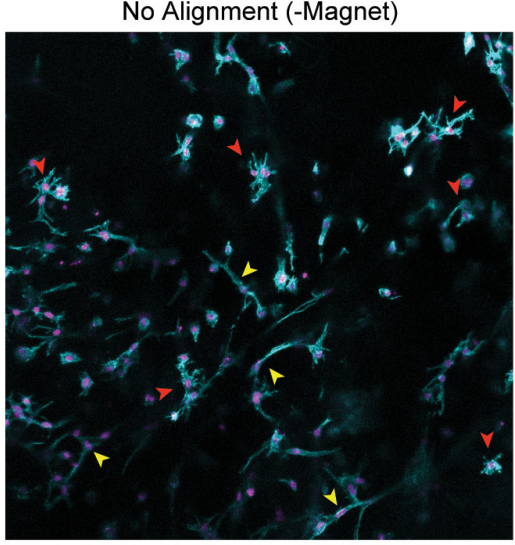

B

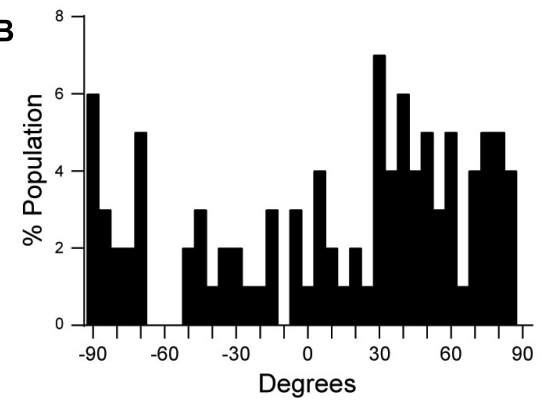

Partial Alignment
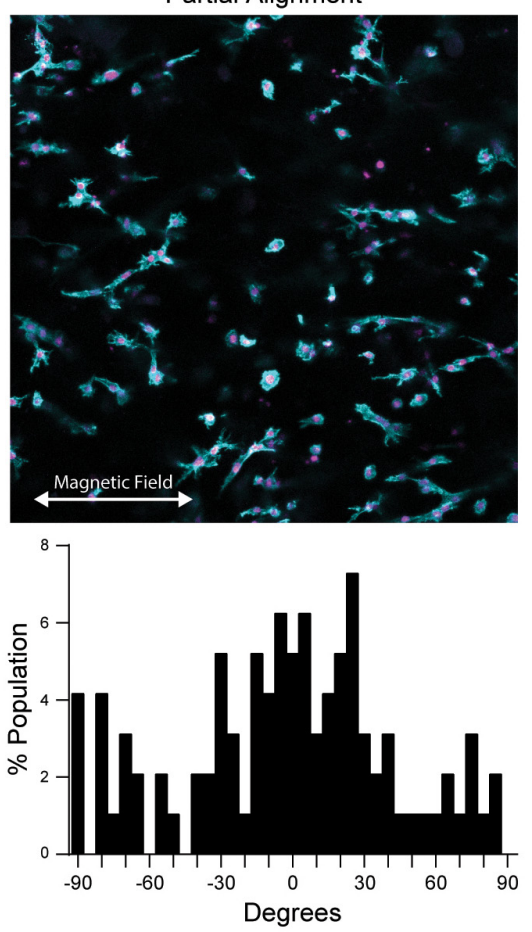
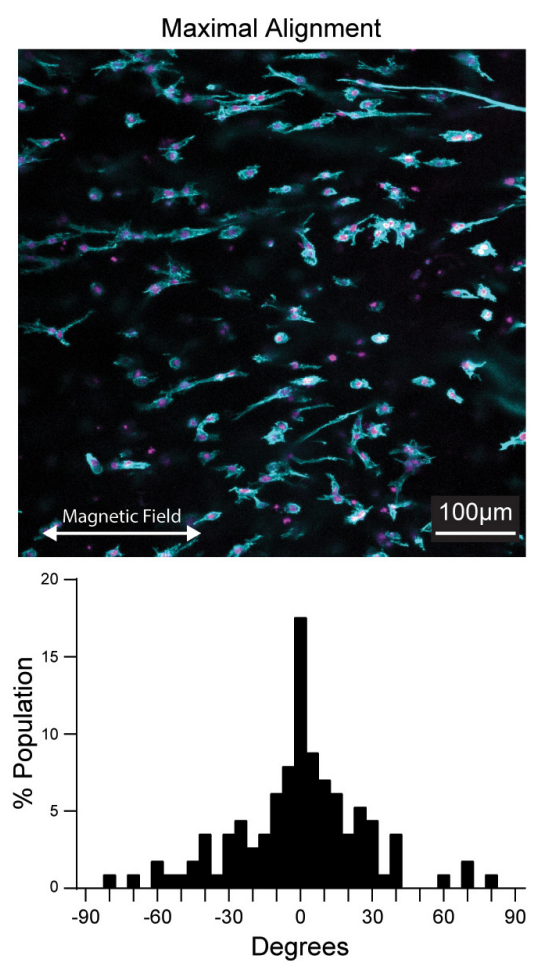

C

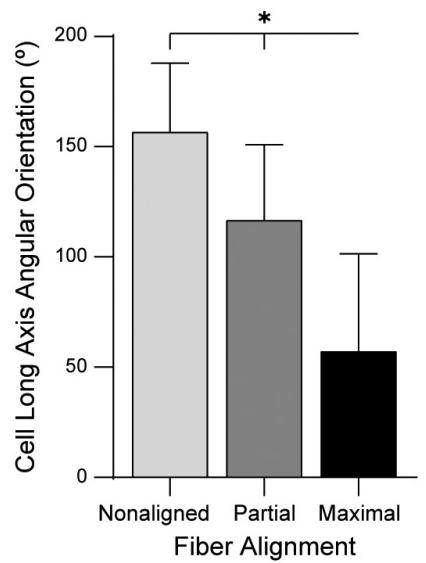

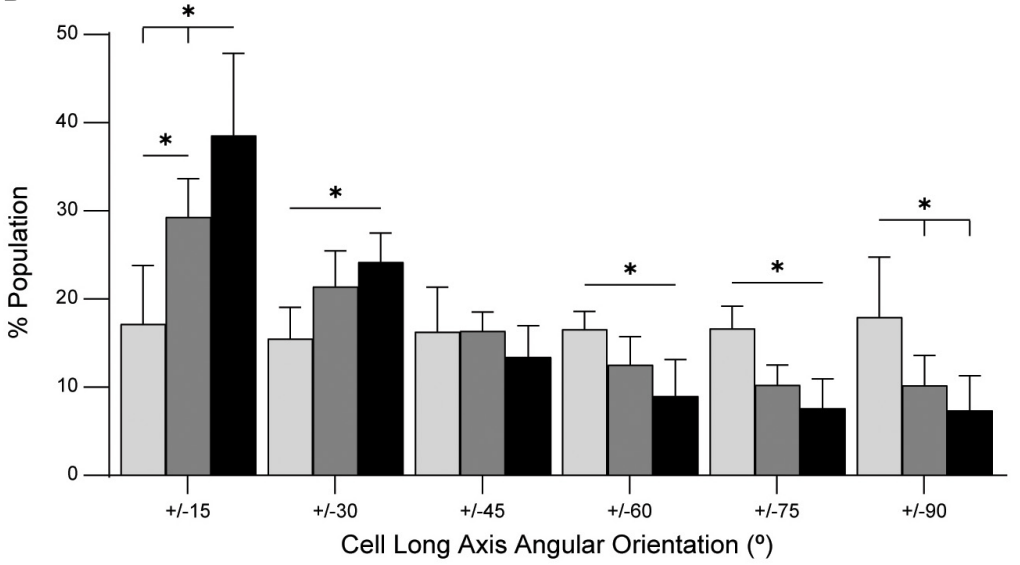

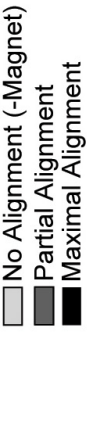

FIGURE 6 | Fiber alignment directs the orientation and morphology of encapsulated tendon fibroblasts. (A) Fluorescent images of primary mouse tendon fibroblasts (tenocytes) cultured in hydrogel composites for 7 days. Red arrowheads indicate stellate morphology cells; yellow arrowheads indicate uniaxially spread cells.

(B) Histograms of cell orientation as a function of fiber alignment. (C) Full width-half max quantification of $\mathrm{n}=10$ cell orientation distributions. (D) Angular stratification of cell orientations as a function of fiber alignment. All data presented as mean $\pm \mathrm{SD}$; *indicates a statistically significant comparison with $p<0.05$.

of fiber alignment was again modulated by magnetic field strength. Migration from spheroids occurred predominantly as multicellular collective strands that contact guided along fiber segments biased in the direction of fiber alignment (Figure 7A and Supplementary Figure 2). Within partially aligned and aligned fibrous matrices, nuclei also appeared elongated in the direction of fiber alignment (Figure 7B). To more directly visualize migration directional bias, we utilized a previously developed custom MATLAB image analysis code to generate heatmap overlays of actin structures (Figure 7C) and rose plots of nuclear locations (Figure 7D) for 25 spheroids (Hiraki et al., 2021). Non-aligned gels promoted radially uniform cell outgrowths and distribution of nuclei. In contrast, the majority of migratory outgrowths in partially aligned and maximally aligned gels occurred within \pm 30 degrees of the axis of fiber alignment. We did not observe a change in the total number of migrating cells across each gel condition, suggesting that fiber alignment does not increase the frequency of cell migration. However, in aligned gels there was an increase in the number of cells migrating as single cells 
or multicellular clusters disconnected from the main body of the spheroid (Figure 7E). The image analysis code also quantifies total migration distance (the summed migration distance of each cell from the spheroid periphery as a measure of net transtromal migration) and maximum invasion depth (the maximal depth into the surrounding stromal matrix of an outgrowth). Collective strands contiguous to the spheroid accounted for the majority of total transtromal migration distance with no significant change as a function of fiber alignment. However, we noted a significant increase in total migration distance of disconnected migratory cells at both levels of fiber alignment (Figure 7F). Despite the emergence of distinct migratory phenotypes, no change in maximum invasion distance was observed across different degrees of fiber alignment or between connected or disconnected phenotypes (Figure 7G). In sum, these data suggest fiber alignment does not increase overall cell migration or migration speed but rather increases directional migration via contact guidance and the frequency of cell-cell breakage events that engender disconnected invasive cell structures.

\section{DISCUSSION}

Here, we describe a means to align magnetic electrospun fibers within a 3D hydrogel composite that models stromal ECM. SPIONs were stably incorporated into DVS fiber segments, enabling control over the density and alignment of fibrous architecture via an externally applied magnetic field. SPION density and magnetic field strength jointly contributed to fiber alignment, enabling fine control over fiber alignment. Fiber entanglement due to the length and density of fiber segments impaired alignment, but shortening fibers via photomasking prevented fiber entanglement during magnetic alignment, thereby increasing the range of achievable fiber densities in $3 \mathrm{D}$ hydrogel composites. We found that both the spreading of individually encapsulated cells and orientation of multicellular migratory structures from spheroids were influenced by the degree of fiber alignment. Aligned fibrous architecture directed uniaxial spreading of primary derived mouse tenocytes in lieu of stellate morphologies. Fiber alignment also biased the direction of multicellular migration from MCF10A spheroids and increased the number of cell-cell breakage events, leading to the emergence of invading single cells and multicellular clusters. While previous methods have been developed to aligned fibers within purified biopolymer hydrogel such as type I collagen, the synthetic fiber-reinforced hydrogel composite system presented here provides more facile orthogonal tuning of fibrous architecture parameters including the degree of alignment, fiber length, and fiber density.

Our custom designed magnetic gelation chamber holds two small N52 neodymium magnets that produce a surface field of $661.9 \mathrm{mT}$. In comparison to previous methods utilizing Teslarange magnetic fields to align type I collagen gels (Dickinson et al., 1994), alignment of SPION-containing DVS fiber segments requires a significantly lower magnetic field strength achievable with small rare earth magnets without the need for specialized or expensive equipment (Figure 2). In comparison to other methods of aligning fibers, such as flow-induced alignment or fibroblastmediated matrix reorganization, control over magnetic fiber fabrication and field strength provide a higher degree of control of fiber alignment. As anticipated, we found fiber alignment to be sensitive to both SPION density within fiber segments and field strength (Figure 3). As such, we were able to tune the degree of fiber alignment within hydrogel composites to produce different degrees of alignment. Enhanced control over the degree of alignment could enable modeling of progressive stages of tissue repair or pathogenesis that involve matrix fibers such as tendon regeneration or invasive ductal carcinomas, respectively (Provenzano et al., 2006; Schoenenberger et al., 2018). Furthermore, the degree of fiber alignment could be tuned to reflect histologic samples or in situ images of tissue to more accurately model specific tissue types or states of disease.

As the stroma possesses a high density of fibrous ECM proteins, we next modulated fiber density within our hydrogel composites and observed fiber entanglement and reduced alignment when the density of fibers exceeded $3 \mathrm{v} / \mathrm{v} \%$. To prevent entanglement, we used chrome photomasks to shorten fibers (Figure 4). Photomasking decreased variance in fiber lengths and enabled increased alignment at higher fiber densities. The large variance in fiber length without photomasking is likely due to the processing of deposited fibers mats into individual fiber segments, which involves vortexing resuspended fiber mats-an uncontrolled process yielding fibers between 100 and $550 \mu \mathrm{m}$ in length. In contrast, photomasking produced more consistent fiber lengths. Alignment of shorter fiber segments did not result in entanglement and proved insensitive to boundary effects (Figure 4G). Fibers near the glass coverslip bottom and sides of the PDMS gasket were aligned to the same degree as fibers within the center of the gel. In comparison to flow-induced fiber alignment, which creates alignment artifacts near rigid boundaries, magnetic alignment readily overcomes initial fiber orientation resulting from the injection of hydrogel precursor solution.

Functionalization of fiber segments with cell-adhesive RGD allowed cells to engage and spread along fiber segments and respond to matrix alignment. We encapsulated primary derived mouse tenocytes into aligned hydrogel composites and observed cell spreading along fiber segments and a morphologic transition from stellate to uniaxial morphologies oriented in the direction of fiber alignment (Figure 6). Alignment of tenocytes has potential implications in tendon wound repair, as the cells and matrix within this tissue are highly organized (Docheva et al., 2015). Given the non-contact nature of this method, alignment of cell-laden hydrogels within in vivo wound sites is an exciting possibility currently under exploration.

Similar to single tenocyte spreading, we observed multicellular migration from MCF10A spheroids biased in the direction of fiber alignment (Figure 7). Cells migrating as collective strands contact guided along fiber segments with nuclei elongated in the direction of fiber alignment. Interestingly, we noted a significant increase in disconnected migratory outgrowths including single cell and multicellular clusters with fiber alignment. This switch in migratory phenotype may indicate directional migration 


\section{A}
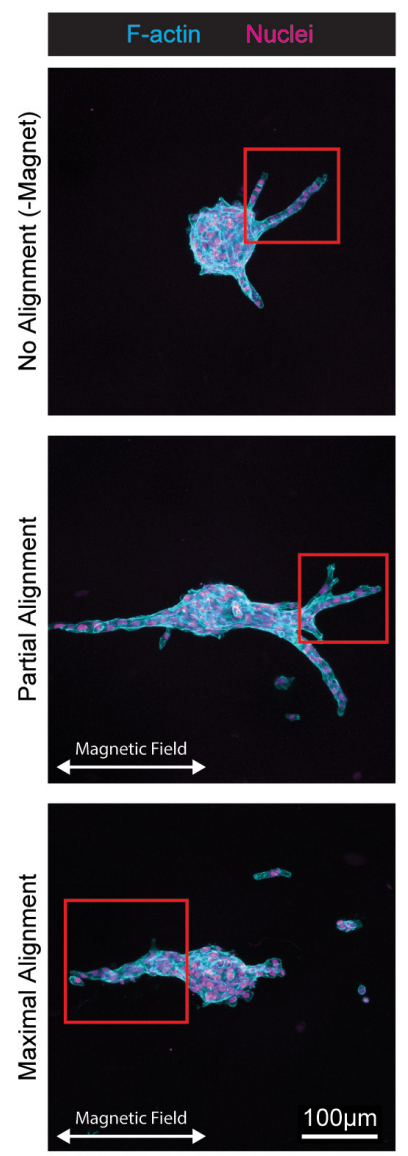

E

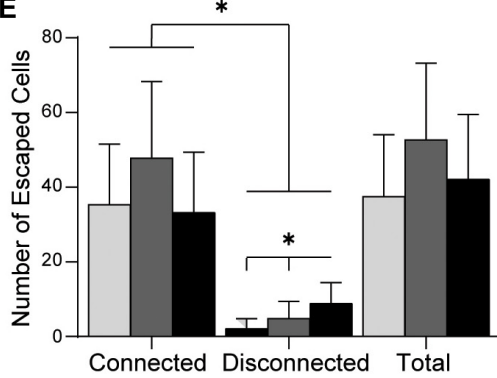

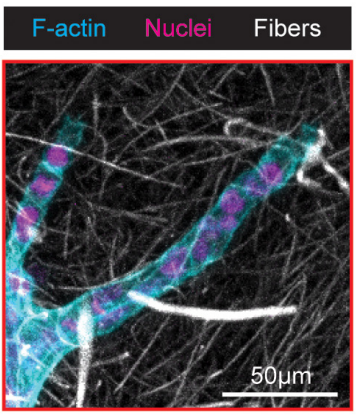
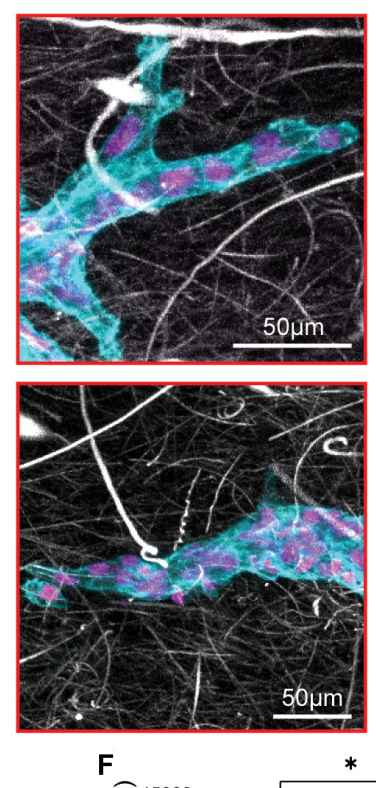

\section{c}
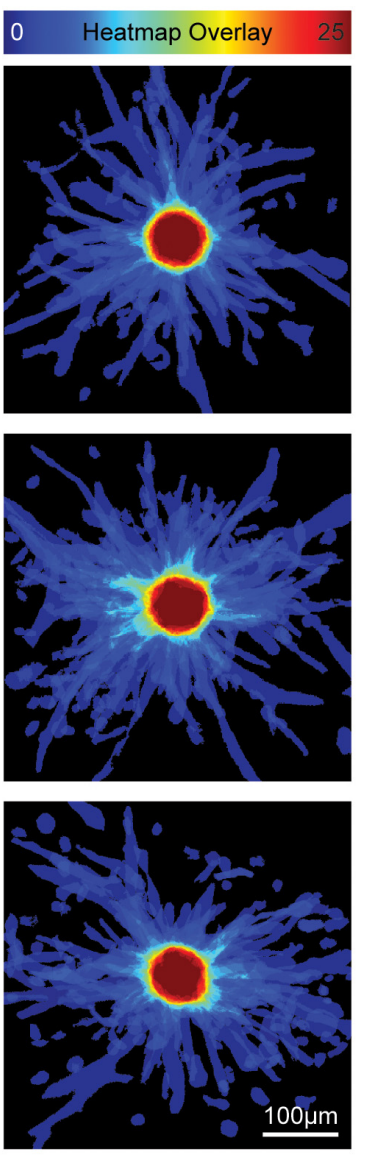

G

D
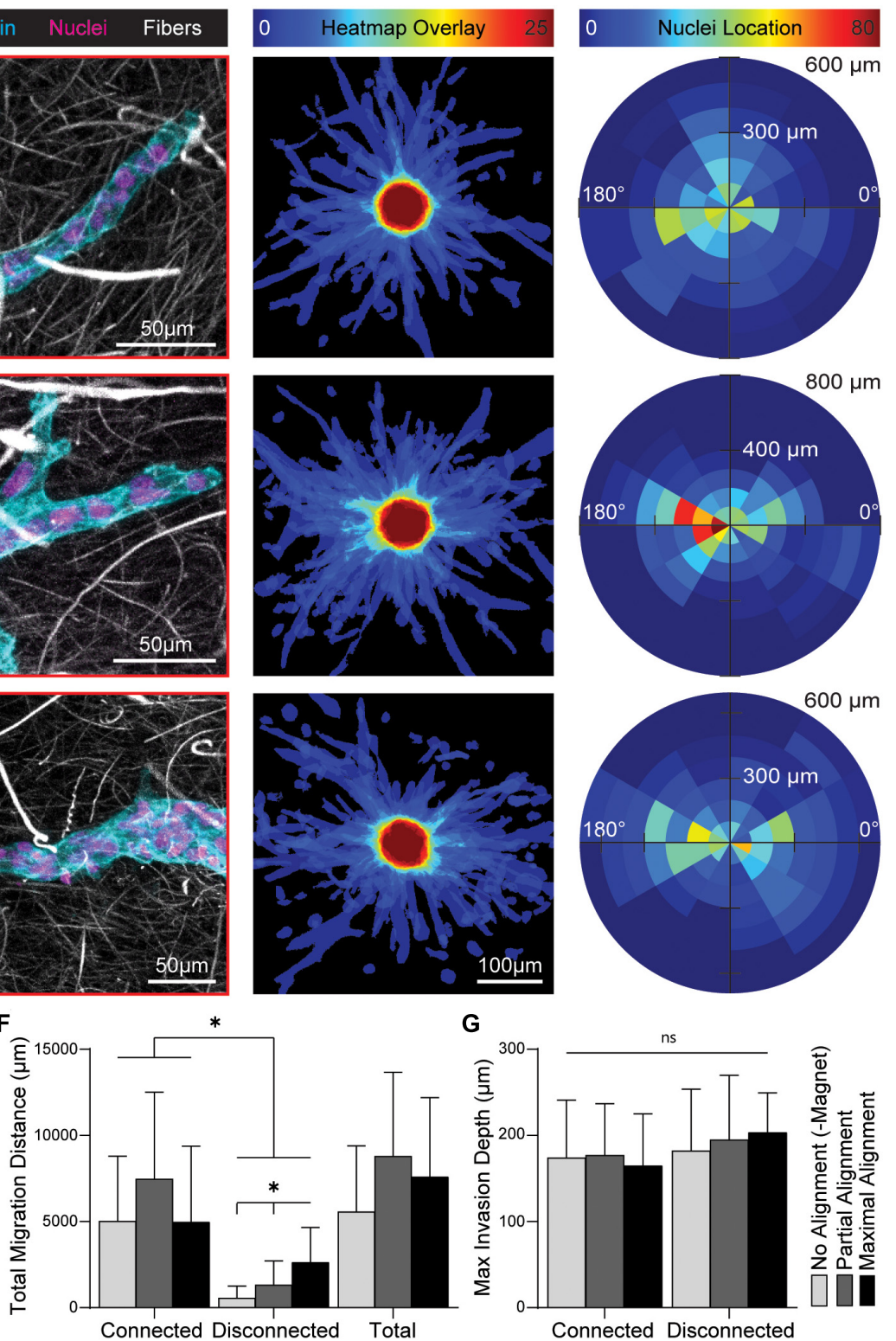

FIGURE 7 | Fiber alignment biases migration direction from multicellular MCF10A spheroids and induces cell-cell breakage events. (A) Fluorescent images of cell outgrowth from multicellular MCF10A spheroids encapsulated in DVS hydrogel composites after 6 days. (B) Higher magnification image including DVS fibers from location depicted by inset in (A). (C) Heatmap overlays created by an aggregate sum of binarized actin channels and (D) rose plots of migratory cell nuclei location for $n=25$ spheroids per condition. Quantification of (E) total number of migratory cells and (F) total migration distance stratified by outgrowth contiguity with the spheroid. (G) Maximum invasion depth of individual outgrowths stratified by contiguity with the spheroid. All data presented as mean \pm SD; *indicates a statistically significant comparison with $p<0.05$.

along aligned matrix fibers promotes EMT signaling and/or decreased cell-cell adhesion to induce cell-cell breakage events (Pearson, 2019). Another explanation is that aligned matrices increase migration speed, causing leading cells to lose adhesion to slower moving trailing cells. Enhanced cell migration speed along aligned fibrous matrices has been reported in 2D settings (Wang et al., 2018; Xue et al., 2018). While we did not observe an increase in net invasion depth with fiber alignment, instantaneous migration speeds were not assessed here. As the bulk DVS hydrogel stiffness/crosslinking is separately defined from fiber density and alignment (Matera et al., 2019; Hiraki et al., 2021), this hydrogel composite can be used to investigate the individual contributions of fiber alignment and hydrogel stiffness/crosslinking on 3D cell migration speed in future studies (Riching et al., 2015; Wang et al., 2018). Further investigation with timelapse imaging could directly assess if fiber alignment 
increases migration speed during proteolysis-dependent 3D cell migration. Orthogonal tuning of fiber density and alignment at a constant hydrogel stiffness could also provide insight into the influence of tumor-associated collagen signatures (TACS), as previously described by Provenzano et al. (2008). TACS describes three major changes in collagen architecture surrounding solid tumors during breast cancer progression that facilitate metastatic invasion, two of which are increased fiber density and radial alignment of fibers at the tumor-stroma interface. By varying input volume fraction of fiber segments and magnetic field strength, matrix fiber density and alignment can be differentially tuned to model progressive states of tumor stroma.

While RGD was used to enable cell adhesion to fiber segments here, other ECM peptides can be used to model full length proteins such as the GXOGER sequence of type I collagen (DePalma et al., 2021). As DVS fiber segments are not hydrolytically or proteolytically degradable, cell force-mediated reorganization of fibrous architecture could also be investigated. The magnetic, electrospun fiber segments developed here can be easily integrated within other natural and synthetic biomaterials. MMP-cleavable DVS hydrogel was selected as the bulk material here due to its tunability of bulk stiffness and crosslinking via Michael-type addition. For integration with other hydrogels, crosslinking kinetics should be carefully taken into account. Fiber segments were immobilized after $8 \mathrm{~min}$ of DVS hydrogel crosslinking via Michael-type addition. The post-gelation degree of fiber alignment is likely a function of magnetic field strength in conjunction with hydrogel precursor solution viscosity as a function of crosslinking. As such, stronger magnets may be required to achieve the same degree of fiber alignment if hydrogel crosslinking kinetics are significantly faster than the DVS hydrogels employed here.

We present a hydrogel composite system consisting of SPIONcontaining electrospun fiber segments which can be aligned within an externally applied magnetic field. We demonstrate orthogonal tunability of key fibrous matrix attributes including fiber length, fiber density, and degree of fiber alignment. The ability to align magnetic fibers proved insensitive to boundary conditions, allowing homogeneous fiber alignment throughout a millimeter-scale hydrogel. With this system, we demonstrate the ability to align single encapsulated primary mouse tenocytes, which may have utility as an injectable biomaterial therapy to mediate tendon repair. Furthermore, we show control over directional multicellular migration from MCF10A spheroids and find that fiber alignment induces breakage events leading to migration phenotype switching from collective strands to

\section{REFERENCES}

Baker, B. M., and Chen, C. S. (2012). Deconstructing the third dimension-how 3D culture microenvironments alter cellular cues. J. Cell Sci. 125, 3015-3024. doi: $10.1242 /$ jcs.079509

Boudaoud, A., Burian, A., Borowska-Wykręt, D., Uyttewaal, M., Wrzalik, R., Kwiatkowska, D., et al. (2014). FibrilTool, an ImageJ Plug-in to Quantify Fibrillar Structures in Raw Microscopy Images. Nat. Protoc. 9, 457-463. doi: 10.1038/nprot.2014.024

Brownfield, D. G., Venugopalan, G., Lo, A., Mori, H., Tanner, K., Fletcher, D. A., and Bissell, M. J. (2013). Patterned collagen fibers orient branching mammary single cells and multicellular clusters. The tunability of fibrous architecture within this hydrogel composite and the ability to integrate magnetic fibers with other biomaterials could enable modeling of stromal tissue architectures for future studies of connective tissue repair and disease processes.

\section{DATA AVAILABILITY STATEMENT}

The raw data supporting the conclusions of this article will be made available by the authors, without undue reservation.

\section{AUTHOR CONTRIBUTIONS}

$\mathrm{HH}$ : first author generating data for fiber alignment, COMSOL modeling, and cell studies. DM: fiber segment fabrication protocol. MR: hydrogel composite fabrication. RK: tenocyte harvesting. CT, MES, AW, and MCS: magnetic gelation chamber fabrication. SD: photomask design and fabrication. AZ: data collection and Fibril Tool quantifications. All authors contributed to the article and approved the submitted version.

\section{FUNDING}

DM acknowledge financial support from the National Science Foundation Graduate Research Fellowship Program (DGE1256260). BB acknowledges financial support from an NIH Pathway to Independence Award (HL124322).

\section{SUPPLEMENTARY MATERIAL}

The Supplementary Material for this article can be found online at: https://www.frontiersin.org/articles/10.3389/fbioe. 2021.679165/full\#supplementary-material

\begin{abstract}
Supplementary Figure 1 | Degree of fiber alignment is determined by both encapsulated SPION density and magnetic field strength. Color maps produced by OrientationJ of fiber alignment at $1 \mathrm{~V} / \mathrm{V} \%$ fiber density in 3D DVS hydrogels across $\mathbf{( A )}$ a range of encapsulated SPION densities and $\mathbf{( B )}$ across a range of magnet spacings.
\end{abstract}

Supplementary Figure 2 | Fiber alignment induces directional migration from MCF10A spheroids. Fluorescent images of cell outgrowth from MCF10A spheroids encapsulated in DVS hydrogel composites after 6 days for various degrees of fiber alignment.

epithelium through distinct signaling modules. Curr. Biol. 23, 703-709. doi: 10.1016/j.cub.2013.03.032

Carey, S. P., Goldblatt, Z. E., Martin, K. E., Romero, B., Williams, R. M., and Reinhart-King, C. A. (2016). Local extracellular matrix alignment directs cellular protrusion dynamics and migration through Racl and FAK. Integr. Biol. 8, 821-835. doi: 10.1039/c6ib00030d

Dallas, S. L., Chen, Q., and Sivakumar, P. (2006). Dynamics of assembly and reorganization of extracellular matrix proteins. Curr. Top. Dev. Biol. 75, 1-24. doi: 10.1016/S0070-2153(06)75001-3

DePalma, S. J., Davidson, C. D., Stis, A. E., Helms, A. S., and Baker, B. M. (2021). Microenvironmental Determinants of Organized IPSC-Cardiomyocyte 
Tissues on Synthetic Fibrous Matrices. Biomater. Sci. 9, 93-107. doi: 10.1039/ d0bm01247e

Dickinson, R. B., Guido, S., and Tranquillo, R. T. (1994). Biased Cell Migration of Fibroblasts Exhibiting Contact Guidance in Oriented Collagen Gels. Ann. Biomed. Eng. 22, 342-356. doi: 10.1007/bf02368241

Docheva, D., Müller, S. A., Majewski, M., and Evans, C. H. (2015). Biologics for Tendon Repair. Adv. Drug Deliv. Rev. 84, 222-239. doi: 10.1016/j.addr.2014. 11.015

Dumont, N., Liu, B., Defilippis, R. A., Chang, H., Rabban, J. T., Karnezis, A. N., et al. (2013). Breast fibroblasts modulate early dissemination, tumorigenesis, and metastasis through alteration of extracellular matrix characteristics. Neoplasia 15, 249-262. doi: 10.1593/neo.121950

Guo, C., and Kaufman, L. J. (2007). Flow and Magnetic Field Induced Collagen Alignment. Biomaterials 28, 1105-1114. doi: 10.1016/j.biomaterials.2006.10. 010

Gutiérrez, L., de la Cueva, L., Moros, M., Mazarío, E., de Bernardo, S., de la Fuente, J. M., et al. (2019). Aggregation Effects on the Magnetic Properties of Iron Oxide Colloids. Nanotechnology 30, 112001. doi: 10.1088/1361-6528/ aafbff

Han, W., Chen, S., Yuan, W., Fan, Q., Tian, J., Wang, X., et al. (2016). Oriented collagen fibers direct tumor cell intravasation. Proc. Natl. Acad. Sci. U.S.A. 40, 11208-11213. doi: 10.1073/pnas.1610347113

Hiraki, H. L., Matera, D. L., Wang, W. Y., Zarouk, A. A., Argento, A. E., Buschhaus, J. M., et al. (2021). Fiber Density and Matrix Stiffness Modulate Distinct Cell Migration Modes in a 3D Stroma Mimetic Composite Hydrogel. bioRxiv [preprint]. doi: 10.1101/2021.02.27.433190

Hong, J., Yeo, M., Yang, G. H., and Kim, G. (2019). Cell-electrospinning and its application for tissue engineering. Int. J. Mol. Sci. 20. doi: 10.3390/ijms2024 6208

Iannone, M., Ventre, M., Formisano, L., Casalino, L., Patriarca, E. J., and Netti, P. A. (2015). Nanoengineered surfaces for focal adhesion guidance trigger mesenchymal stem cell self-organization and tenogenesis. Nano Lett. 15, 15171525. doi: $10.1021 / \mathrm{nl} 503737 \mathrm{k}$

Ingman, W. V., Wyckoff, J., Gouon-Evans, V., Condeelis, J., and Pollard, J. W. (2006). Macrophages promote collagen fibrillogenesis around terminal end buds of the developing mammary gland. Dev. Dyn. 235, 3222-3229. doi: 10. 1002/dvdy.20972

Kim, J. I., Hwang, T. I., Aguilar, L. E., Park, C. H., and Kim, C. S. (2016). A controlled design of aligned and random nanofibers for $3 \mathrm{~d}$ bi-functionalized nerve conduits fabricated via a novel electrospinning set-up. Sci. Rep. 6, 1-12. doi: $10.1038 /$ srep23761

Kirkpatrick, N. D., Andreou, S., Hoying, J. B., and Utzinger, U. (2007). Live imaging of collagen remodeling during angiogenesis. Am. J. Physiol. Heart Circ. 292. doi: 10.1152 /ajpheart.01234.2006

Li, L., Eyckmans, J., and Chen, C. S. (2017). Designer biomaterials for mechanobiology. Nat. Mater. 16, 1164-1168. doi: 10.1038/nmat5049

Matera, D. L., Wang, W. Y., Smith, M. R., Shikanov, A., and Baker, B. M. (2019). Fiber Density Modulates Cell Spreading in 3D Interstitial Matrix Mimetics. ACS Biomater. Sci. Eng. 5, 2965-2975. doi: 10.1021/acsbiomaterials.9b00141

Mason, B. N., Starchenko, A., Williams, R. M., Bonassar, L. J., and Reinhart-King, C. A. (2013). Tuning three-dimensional collagen matrix stiffness independently of collagen concentration modulates endothelial cell behavior. Acta Biomater. 9, 4635-4644. doi: 10.1016/j.actbio.2012.08.007

Ng, C. P., and Swartz, M. A. (2003). Fibroblast Alignment under Interstitial Fluid Flow Using a Novel 3-D Tissue Culture Model. Am. J. Physiol. Heart Circ. Physiol. 284, 1771-1777.

Ng, C. P., and Swartz, M. A. (2006). Mechanisms of Interstitial Flow-Induced Remodeling of Fibroblast-Collagen Cultures. Ann. Biomed. Eng. 34, 446-454. doi: 10.1007/s10439-005-9067-3

Omidinia-Anarkoli, A., Boesveld, S., Tuvshindorj, U., Rose, J. C., Haraszti, T., and De Laporte, L. (2017). An Injectable Hybrid Hydrogel with Oriented Short Fibers Induces Unidirectional Growth of Functional Nerve Cells. Small 13:1702207. doi: $10.1002 /$ smll.201702207

Patil, R. M., Thorat, N. D., Shete, P. B., Bedge, P. A., Gavde, S., Joshi, M. G., et al. (2018). Comprehensive Cytotoxicity Studies of Superparamagnetic Iron Oxide Nanoparticles. Biochem. Biophys. Rep. 13, 63-72.

Pearson, G. W. (2019). Control of Invasion by Epithelial-to-Mesenchymal Transition Programs during Metastasis. J. Clin. Med. 8:646. doi: 10.3390/ jcm8050646
Poltavets, V., Kochetkova, M., Pitson, S. M., and Samuel, M. S. (2018). The role of the extracellular matrix and its molecular and cellular regulators in cancer cell plasticity. Front. Oncol. 8:431. doi: 10.3389/fonc.2018.00431

Provenzano, P. P., Eliceiri, K. W., Campbell, J. M., Inman, D. R., White, J. G., and Keely, P. J. (2006). Collagen Reorganization at the Tumor-Stromal Interface Facilitates Local Invasion. BMC Med. 4:38. doi: 10.1186/1741-7015-4-38

Provenzano, P. P., Inman, D. R., Eliceiri, K. W., Knittel, J. G., Yan, L., Rueden, C. T., et al. (2008). Collagen Density Promotes Mammary Tumor Initiation and Progression. BMC Med. 6:11. doi: 10.1186/1741-7015-6-11

Püspöki, Z., Storath, M., Sage, D., and Unser, M. (2016). Transforms and Operators for Directional Bioimage Analysis: a Survey. Adv. Anat. Embryol. Cell Biol. 219, 69-93. doi: 10.1007/978-3-319-28549-8_3

Ray, A., Slama, Z. M., Morford, R. K., Madden, S. A., and Provenzano, P. P. (2017). Enhanced directional migration of cancer stem cells in 3D aligned collagen matrices. Biophys. J. 112, 1023-1036. doi: 10.1016/j.bpj.2017.01.007

Riching, K. M., Cox, B. L., Salick, M. R., Pehlke, C., Riching, A. S., Ponik, S. M., et al. (2015). 3D Collagen Alignment Limits Protrusions to Enhance Breast Cancer Cell Persistence. Biophys. J. 107, 2546-2558. doi: 10.1016/j.bpj.2014.10.035

Rose, J. C., Gehlen, D. B., Haraszti, T., Köhler, J., Licht, C. J., and De Laporte, L. (2018). Biofunctionalized aligned microgels provide 3D cell guidance to mimic complex tissue matrices. Biomater 163, 128-141. doi: 10.1016/j.biomaterials. 2018.02.001

Rose, J. C., Cámara-Torres, M., Rahimi, K., Köhler, J., Möller, M., De Laporte, L., et al. (2017). Nerve Cells Decide to Orient inside an Injectable Hydrogel with Minimal Structural Guidance. Nano Lett. 17, 3782-3791. doi: 10.1021/acs. nanolett.7b01123

Schoenenberger, A. D., Foolen, J., Moor, P., Silvan, U., and Snedeker, J. G. (2018). Substrate Fiber Alignment Mediates Tendon Cell Response to Inflammatory Signaling. Acta Biomater. 71, 306-317. doi: 10.1016/j.actbio.2018.03.004

Shimada, A., Wada, S., Inoue, K., Ideno, H., Kamiunten, T., Komatsu, K., et al. (2014). Efficient Expansion of Mouse Primary Tenocytes Using a Novel Collagen Gel Culture Method. Histochem. Cell Biol 142, 205-215. doi: 10.1007/ s00418-014-1191-4

Taufalele, P. V., VanderBurgh, J. A., Muñoz, A., Zanotelli, M. R., and ReinhartKing, C. A. (2019). Fiber alignment drives changes in architectural and mechanical features in collagen matrices. PLOS ONE 14:e0216537. doi: 10.1371/ journal.pone. 0216537

Wang, W. Y., Pearson, A. T., Kutys, M. L., Choi, C. K., Wozniak, M. A., Baker, B. M., et al. (2018). Extracellular Matrix Alignment Dictates the Organization of Focal Adhesions and Directs Uniaxial Cell Migration. APL Bioeng. 2:46107. doi: 10.1063/1.5052239

Wakuda, Y., Nishimoto, S., Suye, S. I., and Fujita, S. (2018). Native collagen hydrogel nanofibres with anisotropic structure using core-shell electrospinning. Sci. Rep. 8:6248. doi: 10.1038/s41598-018-24700-9

Wolf, K., Alexander, S., Schacht, V., Coussens, L. M., von Andrian, U. H., van Rheenen, J., et al. (2009). Collagen-based cell migration models in vitro and in vivo. Semin. Cell Dev. Biol. 20, 931-941. doi: 10.1016/j.semcdb.2009.08.005

Xu, Y., Yin, H., Chu, J., Eglin, D., Serra, T., Docheva, D., et al. (2021). An Anisotropic Nanocomposite Hydrogel Guides Aligned Orientation and Enhances Tenogenesis of Human Tendon Stem/Progenitor Cells. Biomater. Sci. 9, 1237-1245. doi: 10.1039/d0bm01127d

Xue, J., Wu, T., and Xia, Y. (2018). Perspective: aligned Arrays of Electrospun Nanofibers for Directing Cell Migration. APL Mater. 6:120902. doi: 10.1063/ 1.5058083

Zhi, X., Fang, H., Bao, C., Shen, G., Zhang, J., Wang, K., et al. (2013). The Immunotoxicity of Graphene Oxides and the Effect of PVP-Coating. Biomaterials 34, 5254-5261. doi: 10.1016/j.biomaterials.2013.03.024

Conflict of Interest: The authors declare that the research was conducted in the absence of any commercial or financial relationships that could be construed as a potential conflict of interest.

Copyright (ㄷ 2021 Hiraki, Matera, Rose, Kent, Todd, Stout, Wank, Schiavone, DePalma, Zarouk and Baker. This is an open-access article distributed under the terms of the Creative Commons Attribution License (CC BY). The use, distribution or reproduction in other forums is permitted, provided the original author(s) and the copyright owner(s) are credited and that the original publication in this journal is cited, in accordance with accepted academic practice. No use, distribution or reproduction is permitted which does not comply with these terms. 